ARTICLE

\title{
Epithermal Neutron Response on Moderated Neutron Spectrometer with Multi-Resonance Filters
}

\author{
Hiroki TSUJI ${ }^{1, *}$, Shigetaka MAEDA ${ }^{1}$, Hideki TOMITA ${ }^{1}$, Jun KAWARABAYASHI ${ }^{1}$, \\ Tetsuo IGUCHI ${ }^{1}$, Tetsuro MATSUMOTO ${ }^{2}$, Jun-ichi HORI ${ }^{3}$ \\ ${ }^{1}$ Department of Quantum Engineering, Graduate School of Engineering, Nagoya University \\ ${ }^{2}$ Advanced Industrial Science and Technology \\ ${ }^{3}$ Kyoto University Research Reactor Institute
}

\begin{abstract}
Precise estimation of neutron spectra in epithermal energy region is quite important for neutron activation analysis in nuclear reactor decommissioning and also for planning the treatments to control patient radiation dose in BNCT. However, conventional moderated neutron spectrometer systems are not so suitable for this purpose. Therefore, we developed a new moderated neutron spectrometer combined with multi-resonance filters. A position sensitive He-3 proportional counter (PSPC) with a neutron moderator and thermal neutron absorber is covered by some neutron resonance filtering materials. Since these filters have specific resonance peaks around the epithermal energy range, a series of response functions with characteristic energy dependence on the incident epithermal neutrons can be obtained along the PSPC.

We measured the response function of a prototype to the pulsed epithermal neutron source at KURRI-LINAC. The prototype system consisted of a He-3 proportional counter with cadmium cover as the thermal neutron absorber and silver plate as the resonance filter. In the experiment, the dips due to the resonance peaks of silver were clearly observed in the response function. It has been also confirmed that the measured response function is in good agreement with the calculated one based on the Monte-Carlo neutron transport code MCBEND.
\end{abstract}

\section{KEYWORDS: epithermal-neutron, clearance level inspection, BNCT, neutron spectrometer, resonance filter, MCBEND}

\section{Introduction}

A number of nuclear reactors have been operated in many countries for more than 30 years. A decommissioning of these aged nuclear reactors begins to be carried out. At the decommissioning process, it is a critical problem to discard building structures which consist of high activation materials (e.g. reactor containment vessel) and low activation ones (e.g. exterior wall). If all materials are treated as low-middle level radioactive wastes, disposal cost becomes huge because geologic disposal is required for the radioactive wastes. At the actual decommissioning, the cost will be added to that by treating non-radioactive wastes as normal factory wastes. ${ }^{1)}$ In process of clearance level inspection, the estimating activation level of the structures is very important. For the estimation, the radioactivity level measurement of actual samples or the activation calculation must be carried out. In activation calculation, an epi-thermal neutron spectrum in building under operation is required, but the accuracy is poor of the neutron energy spectrum obtained either by the Monte Carlo calculations or by Bonner Ball detector measurements. This problem will result in excessive costs. Therefore, a neutron spectrometer is requested which can measure epi-thermal neutron spectrum in building under online operation with a high

*Corresponding Author, E-mail:tsuji@avocet.nucl.nagoya-u.ac.jp degree of accuracy.

In addition, Boron Neutron Capture Therapy (BNCT) with boron compounds including B-10 has been enforced for aiming at cancer therapy. ${ }^{2)}$ Because enforcement of $\mathrm{BNCT}$ requires thermal neutron irradiation to cells, in early phase of BNCT, thermal neutron was irradiated after exposing the tumors by craniotomy procedure. However, in these days, the enforcement of BNCT with no surgical exploration has increased by epi-thermal neutron irradiation from outside the body using neutron moderation in human body. In BNCT, neutron has been irradiated with well planned treatment that is determined by the position of the tumors, the dosage of boron compounds and the irradiated neutron spectrum. ${ }^{3)}$ Actually irradiated neutron spectrum, however, is not necessarily corresponding to assumption value. This problem causes decrease of certainty of BNCT. Therefore, an improvement is required in online measurement of neutron spectrum under irradiating with a high degree of accuracy.

\section{Multi resonance filter method}

A multi resonance filters method proposed by this study utilizes resonance peaks in neutron capture cross-section of certain elements. For an example shown in Fig. 1, silver gives a resonance peak of neutron capture cross-section at 


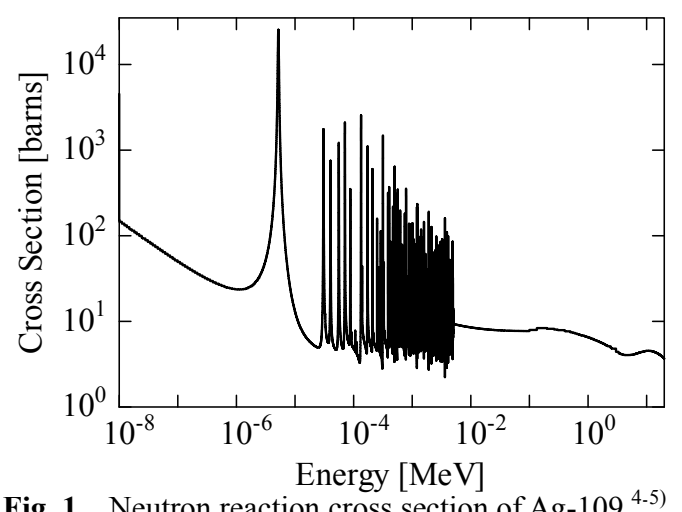

Fig. 1 Neutron reaction cross section of Ag-109. ${ }^{4-5)}$ The resonance peak exists between $10^{-6}$ and $10^{-5} \mathrm{MeV}$.

about $10 \mathrm{eV}$. More elements that have the resonance peak at deferent energy are used as the resonance filter. A detector system with these filters is shown in Fig. 2. A position sensitive ${ }^{3} \mathrm{He}$ proportional counter is placed in center which is surrounded by polyethylene (neutron moderator), $\mathrm{B}_{4} \mathrm{C}$ (thermal neutron absorber) and resonance filters (Silver, Cobalt, Tungsten, Molybdenum, Manganese, Sodium). The thermal neutron absorber is employed to suppress a deviation of detector response between different filters at below $1 \mathrm{eV}$ neutrons. The energy and the absolute value of main resonance peak of the resonance filters are listed in Table 1. Response functions of the detector system calculated by Monte Carlo simulation code "MCBEND"6) are shown in Fig. 3. Resonance dips on the response functions are formed by resonance absorption of the filters. In addition, Fig. 4 shows the difference between the response functions with and without the resonance filters. This response is similar to delta function, so that accuracy of epi-thermal neutron spectrum estimation can be much improved through some unfolding technique.

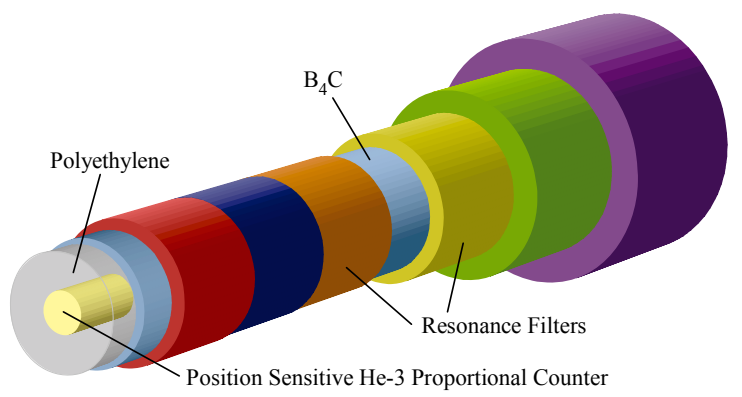

Fig. 2 Concept of the detector with resonance filters

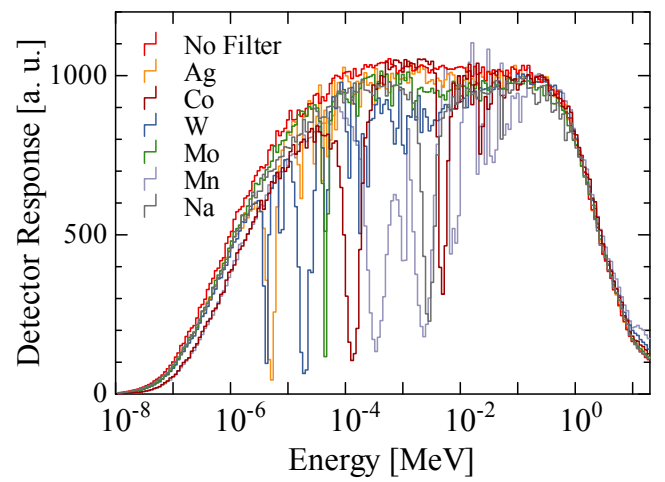

Fig. 3 Response functions of proposed detector calculated by MCBEND

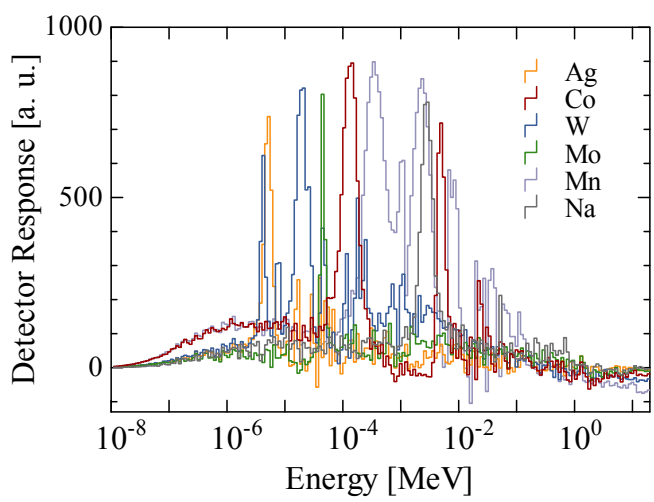

Fig. 4 Results of the response functions with resonance filters subtracted from those with no filter

\section{Evaluation experiments of prototype detector response to epi-thermal neutron}

Experiments to obtain response functions of prototype detector were carried out at KURRI-Linac (Kyoto University Research Reactor Institute - Linear Accelerator) to demonstrate validity of the spectrometer concept. The Linac uses tantalum as heavy metal target, and produces neutrons by photonuclear reactions with gamma rays generated by bremsstrahlung of Linac electrons. Energy distribution of produced neutron has a peak at $1 \mathrm{MeV}$, therefore, epi-thermal neutron beam was obtained with neutron moderator (light water) around the Tantalum target.

First, as shown in Fig. 5, we obtained Time-of-Flight spectrum of the moderated Linac neutrons, where neutron detection signals were generated by B-10 - BGO detector system, which consisted of a ${ }^{10} \mathrm{~B}$ cube located in center of a $\mathrm{BGO}$ detector. Neutrons arriving time was measured by detection of a gamma ray produced by ${ }^{10} \mathrm{~B}(\mathrm{n}, \gamma){ }^{11} \mathrm{~B}$ reaction

Table 1 List of main resonance peak of the resonance filters ${ }^{4-5)}$

\begin{tabular}{|c|c|c|c|c|c|c|}
\hline & $\begin{array}{c}\text { Silver } \\
(\text { Ag-109) } \\
\end{array}$ & $\begin{array}{c}\text { Cobalt } \\
(\text { Co-59) } \\
\end{array}$ & $\begin{array}{l}\text { Tungsten } \\
\text { (W-186) }\end{array}$ & $\begin{array}{c}\text { Molybdenum } \\
\text { (Mo-95) }\end{array}$ & $\begin{array}{c}\text { Manganese } \\
(\mathrm{Mn}-55)\end{array}$ & $\begin{array}{l}\text { Sodium } \\
\text { (Na-23) } \\
\end{array}$ \\
\hline Energy [MeV] & $5.17 \times 10^{-6}$ & $1.31 \times 10^{-4}$ & $1.88 \times 10^{-5}$ & $4.49 \times 10^{-5}$ & $3.37 \times 10^{-4}$ & $2.82 \times 10^{-3}$ \\
\hline Absolute Value [barns] & 25112 & 9740 & 104431 & 12258 & 3288 & 378 \\
\hline
\end{tabular}




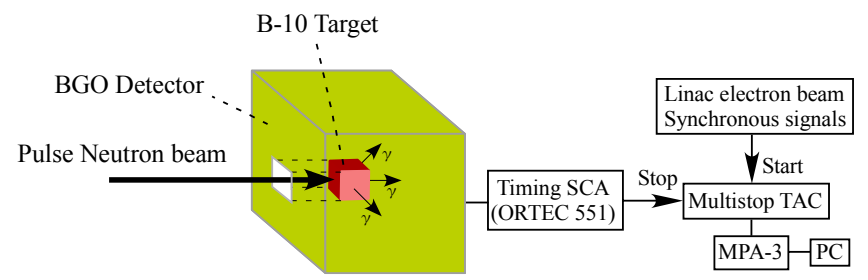

Fig. 5 Experimental system using B-10 - BGO detector

in BGO detector. Fig. 6 represents the obtained TOF spectrum which converted to in energy scale.

A prototype detector system shown in Fig. 7 was assembled, which is composed of a ${ }^{3} \mathrm{He}$ proportional counter (CANBERRA 65NH45), surrounded by a cadmium sheet as thermal neutron absorber and resonance filters (silver, cobalt, tungsten). The Time-of-Flight spectrum was obtained with the same B-10 - BGO signal processing system. The TOF spectrum was converted into energy spectrum, and by comparing it with Linac neutron spectrum, the response functions of the prototype detector system were obtained. The measured response functions are shown in Fig. 8, and show clear resonance dips in the epi-thermal region. Furthermore, response functions of the same system calculated by "MCBEND" are shown in Fig. 9. Shapes of the response functions agree well with each other and validity of the spectrometer concept was mostly confirmed.

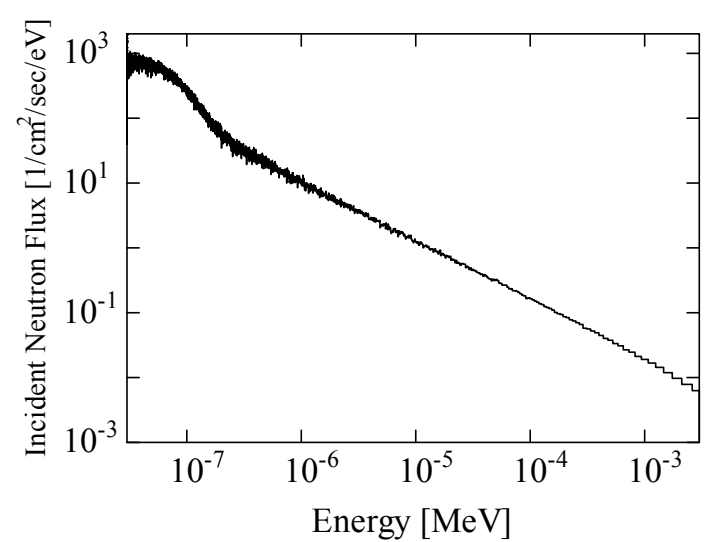

Fig. 6 Energy spectrum of KURRI-Linac neutron beams in this experiment

\section{Conclusions}

High accuracy estimation of the structure activation level and the BNCT epi-thermal neutron spectrum has been requested. As a solution, moderation / resonance type neutron spectrometer concept was proposed and a prototype detector system was demonstrated. By the response function form, the spectrometer concept using multi resonance filters method may be applicable to estimating the epi-thermal neutron dose with a high degree of accuracy compared with conventional method (Bonner Ball detector). In addition, to confirm a validity of the spectrometer concept, experiments aiming at acquisition of response function of prototype

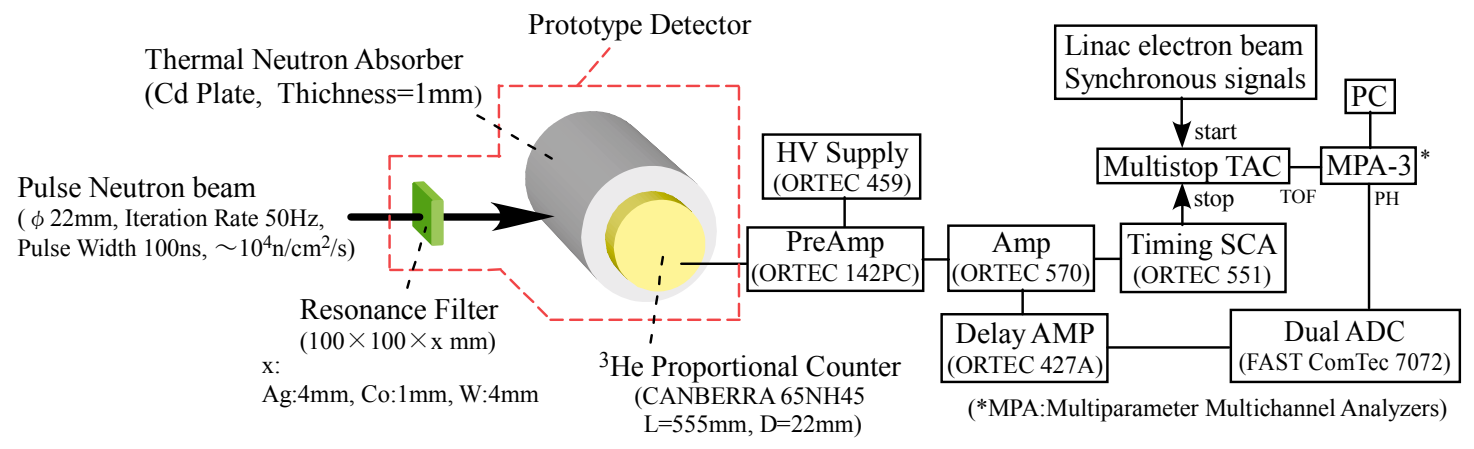

Fig. 7 Experimental system using prototype detector

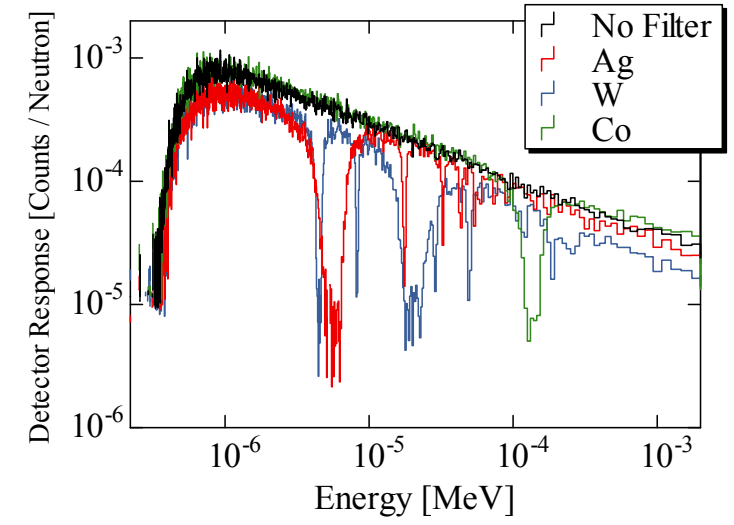

Fig. 8 Response function of prototype detector obtained by experiment

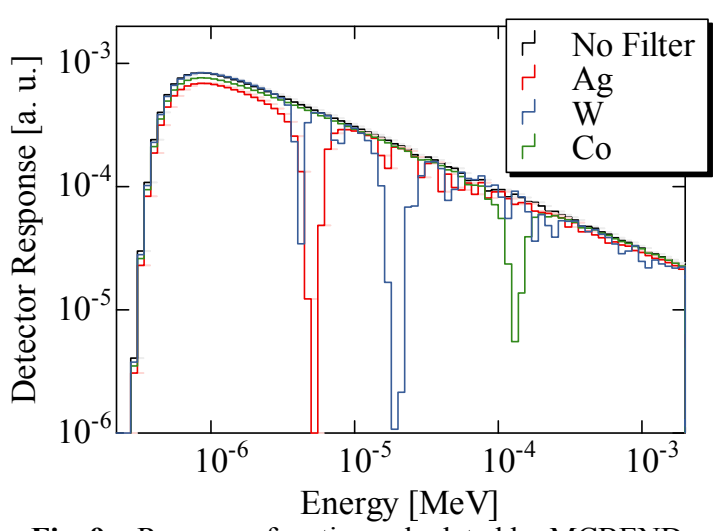

Fig. 9 Response function calculated by MCBEND 
detector were carried out at KURRI-Linac. As the results, obtained response functions had resonance dips and mostly agreed with simulation results. Thus, expected performance of the present spectrometer concept has been successfully demonstrated.

\section{References}

1) Nuclear Safety Commission of Japan, "http://www.nsc.go.jp/haiki/page5/010716_2.pdf”, (2001) [in Japanese]
2) Coderre J.A., Morris G.M.: "The radiation biology of boron neutron capture therapy", Radiat Res, 151, 1, (1999).

3) H. Kumada, et al.: "Improvement of dose calculation accuracy for BNCT dosimetry by the multi-voxel method in JCDS", Applied Radiation and Isotopes, 61, 1045, (2004).

4) National Nuclear Data Center: "http://www.nndc.bnl.gov/ exfor/endf00.jsp", ENDF/B-VII, (2006)

5) P. Oblozinsky, et al.: ENDF/B-VII.0: "Next Generation Evaluated Nuclear Data Library for Nuclear Science and Technology", Nuclear Data Sheets, 107, 2931, (2006)

6) Chucas, S. J., et al.: "Preparing the Monte Carlo Code MCBEND for the 21st Century", Proc. 8th Int. Conf. on Radiation Shielding, Arlington, Texas, (1994). 